\title{
Electronic Properties of Multi-Ablooms Flower-Like Quantum Dots: a 2- D Finite Element Study
}

mehrzad ghorbani ( $\nabla$ mehrzadghorbany@gmail.com )

Qom University of Technology https://orcid.org/0000-0003-0912-7203

\section{Research Article}

Keywords: 2-D multi-abloom nanoflower, 2-D finite element and bilinear method, Galerkin method, electronic properties

Posted Date: April 16th, 2021

DOl: https://doi.org/10.21203/rs.3.rs-394408/v1

License: (9) This work is licensed under a Creative Commons Attribution 4.0 International License. Read Full License 


\title{
Electronic properties of Multi-ablooms Flower-Like Quantum Dots:
}

\section{a 2- D Finite Element Study}

\author{
Mehrzad Ghorbani ${ }^{+}$
}

${ }^{+}$Department of Mathematics, Qom University of Technology, Qom, Iran.

Email: ghorbani@qut.ac.ir, mehrzadghorbany@gmail.com

\begin{abstract}
In this paper, we investigated the electronic properties of two dimensional multiabloom nanoflower. Also, we approximated a typical form of the Schrödinger equation through an appropriate 2-D finite element approach, and then, we have investigated the effects of different parameters such as number of ablooms, number of rose, variety geometric domain shape and systems size on the energy levels, eigenfunctions, and dipole matrix elements.
\end{abstract}

Keywords: 2-D multi-abloom nanoflower, 2-D finite element and bilinear method, Galerkin method, electronic properties.

\section{Introduction}

Because of vast applications in science and technologies, during last few years, quantum dots (QDs) have been an appealing field study. During last few years, a wide variety of shapes, such as quantum dots arrays [1], conical [2], rectangular[3], cylindrical [4], disc-shaped [5], elliptical [6], lens-shaped [7], pyramidal [8], spherical [9], T-shaped [10], Y-shaped [11], etc., for the quantum dot systems have been studied. But as we know up to now, the electronic inter-sub-band structures of nanoflower systems [25] has not so far been addressed. It is clear that due to the complicated form of the quantum dot shapes, the calculation of energy levels and wave functions, analytically, is a nontrivial task [12].

In order to study these quantum dots different methods such as variational method [13, 35], 1/N expansion method [14], quantum cellular automata [15], density functional calculations [16], exact diagonalization [17], finite difference technique [18,36], selfconsistent Hartree method [18], k.p theory [19], Monte Carlo [20], molecular dynamics 
[21], Green's functions [22, 37], Nikiforov-Uvarov method [23], Transfer matrix method [24] etc. have so far been used.

The finite element method (FEM) is a numerical technique for solving PDEs and was originally applied to problems in engineering, structural mechanics applied sciences and are effective for problems with nonregular and complicated domain geometry. In this method, there is a natural variational approach to the numerical solution of elliptic boundary value problems (BVP) such as Helmholtz form of the Schrodinger, which minimize residual over some suitable finite-dimensional subspace defined on the elements.

The FEM involves four basic steps: first, we must partition the solution region of the PDE into a finite number of nonoverlap elements which the rectangular or triangular shapes and $h$ or $p$ refining are most common for planar and 2-D problems. The collection of all regular or congruent elements should cover and resemble the original region as closely as possible. In the second step, we derive interpolation and governing equations for a typical element by defining a complete polynomial on an element. The smooth degree of assembling elements, determine continuity of global approximation. This step, determine blocks and entries of the problem coefficient stiffness matrix. In the third step, we need assemble all partition elements of the solution region to obtain the global coefficient matrix. Finally, the resulting sparse system of equations will be solved.

Here and in our study, the given polygon region divided into 361 uniform rectangular elements. [29,30,31,32,33,34]

In the present work, we have solved a typical 2-D Schrodinger equation by means of the FEM. We have evaluated the energy eigenvalues and corresponding eigenfunctions of a multi-abloom nano-flower. Then we have computed the corresponding dipole matrix elements $M_{12}$ that is an important parameter in evaluation of optical properties of the semiconducting system through density matrix approach.

\section{Finite Element Solution of the 2-D Schrödinger Eq.}

In the envelop function approach, we assume a 2-D Schrödinger equation as, 


$$
\begin{aligned}
H \psi(x, y) & =\frac{\mathrm{h}^{2}}{2 m^{*}}\left(\frac{d^{2}}{d x^{2}}+\frac{d^{2}}{d y^{2}}\right) \psi(x, y)+V(x, y) \psi(x, y) \\
& =\frac{\mathrm{h}^{2}}{2 m^{*}} \Delta \psi(x, y)+V(x, y) \psi(x, y) \\
& =\alpha \Delta \psi(x, y)+V(x, y) \psi(x, y) \\
& =E \psi(x, y)
\end{aligned}
$$

In fact, this 2-D time independent Schrödinger equation is a linear eigenvalue problem with Dirichlet boundary conditions (BCs). The problem domain is rectangular $\Omega=\left[a_{x}, b_{x}\right] \times\left[a_{y}, b_{y}\right]$ where in the normalized case, this domain can be scaled and transformed to the form $\Omega=[0,1] \times[0,1]$. In equation (1), h, $\mathrm{m}^{*}$ and $V(x, y)$ are the Plank constant, effective mass and geometrical confining potential respectively and also set $\alpha=\frac{\mathrm{h}^{2}}{2 m^{*}}$.

As explained, in the FEM, we discretize domain space into some rectangular element subdomains and build bilinear base functions. If we assume $n_{x}$ and $n_{y}$ number of discretization points along $x$ and $y$ direction axes, the steps along the $x$ and $y$ directions will be $h_{x}=\frac{1}{n_{x}}, h_{y}=\frac{1}{n_{y}}$. Then, we have $0=x_{0}<x_{1}<\ldots<x_{n_{x}}=1$, $0=y_{0}<y_{1}<\ldots<y_{n_{y}}=1, \quad x_{i}=a_{x}+i h_{x}, \quad y_{i}=a_{y}+j h_{y}$. The bilinear polynomial basis functions defined on the rectangular element can be written as,

$$
\varphi_{i, j}(x, y)= \begin{cases}\frac{x_{i+1}-x}{x_{i+1}-x_{i}} \frac{y_{j+1}-y}{y_{j+1}-y_{j}}, & x_{i} \leq x \leq x_{i+1}, y_{j} \leq y \leq y_{j+1} \\ \frac{x_{i-1}-x}{x_{i-1}-x_{i}} \frac{y_{j+1}-y}{y_{j+1}-y_{j}}, & x_{i-1} \leq x \leq x_{i}, y_{j} \leq y \leq y_{j+1} \\ \frac{x_{i-1}-x}{x_{i-1}-x_{i}} \frac{y_{j-1}-y}{y_{j-1}-y_{j}}, & x_{i-1} \leq x \leq x_{i}, y_{j-1} \leq y \leq y_{j} \\ \frac{x_{i+1}-x}{x_{i+1}-x_{i}} \frac{y_{j-1}-y}{y_{j-1}-y_{j}}, & x_{i} \leq x \leq x_{i+1}, y_{j-1} \leq y \leq y_{j} \\ 0 \quad & \text { Otherwise }\end{cases}
$$

where $i=0,1,2, \ldots, n_{x}$ and $j=0,1,2, \ldots, n_{y}$. The total number of points are equal $\left(1+n_{x}\right)\left(1+n_{y}\right)$. Since the wave functions degenerate on the boundaries, the boundary 
nodes delete from computing and are not necessary. Therefore, the number of unknown variables (and hence the number of algebraic equations) are equal to number of internal discretization points $\left(n_{x}-1\right)\left(n_{y}-1\right)$.

Now, we construct the finite element approximation of the wave-function through linear combination of basis functions Eq. (2) on all internal nodes,

$\varphi(x, y)=\sum_{i=1, j=1}^{n_{x}-1, n_{y}-1} u_{i, j} \varphi_{i, j}(x, y)$

where $u_{i, j}$ are unknowns. For simplicity, we use normalized and iso-parametric elements by scaling $\xi=\frac{x-x_{i}}{h_{x}}$ and $\eta=\frac{y-y_{j}}{h_{y}}$. Therefore, we can rewrite the equation (2) as an alternative,

$$
\varphi_{i, j}(\xi, \eta)=\left\{\begin{array}{l}
(1-\xi)(1-\eta), 0 \leq \xi \leq 1,0 \leq \eta \leq 1 \\
(1+\xi)(1-\eta),-1 \leq \xi \leq 0,0 \leq \eta \leq 1 \\
(1+\xi)(1+\eta),-1 \leq \xi \leq 0,-1 \leq \eta \leq 0 \\
(1-\xi)(1+\eta), 0 \leq \xi \leq 1,-1 \leq \eta \leq 0 \\
0 \quad, \quad \text { Otherwise }
\end{array}\right.
$$

Since, in the vicinity of the point $\left(x_{i}, y_{j}\right)$ there are only 9 neighborhood points and naturally 4 proper related elements, thus in matrix form of the Hamiltonian, there are only 9 non-zero diagonal layers of elements and we will have a resulting tri-diagonal block Hamiltonian matrix. Each of these blocks is 3 by 3 and they are symmetric. Now, we define residual through substituting the linear expansion (3) into the equation (1),

$$
R(x, y)=\alpha \Delta \varphi)(x, y)+V(x, y) \psi /(x, y)-E \psi /(x, y), \quad x \in \Omega
$$

The approximated wave-function $\varphi((x, y)$ is in the generalized and distributional form [31], thus we can use the Galerkin weighted residual method as,

$$
\iint_{\Omega} R(x, y) \varphi_{i, j}(x, y) d x d y=0
$$

where, in a more precise manner we have,

$$
\int_{y_{j-1}}^{y_{j+1}} \int_{x_{i-1}}^{x_{i+1}} R(x, y) \varphi_{i, j}(x, y) d x d y=0,
$$

And in the normalized space we have, 
$\int_{-1}^{1} \int_{-1}^{1} R(\xi, \eta) \varphi_{i, j}(\xi, \eta) d \xi d \eta=0$

In order to evaluate the equations (6), we need to use green theorem of second kind as $[31,34]$,

$\iint_{\Omega} \Phi \Delta U d \Omega+\iint_{\Omega} \nabla \Phi g \nabla U d \Omega=\iint_{\Gamma} \Phi \frac{\partial U}{\partial n} d \Gamma$

Due to homogeneous Dirichlet boundary condition, this equation can be simplified into,

$\iint_{\Omega} \Phi \Delta U d \Omega=-\iint_{\Omega} \nabla \Phi g \nabla U d \Omega$

Figure (1) represents a simple description about the relating basis functions in the neighborhood of the point $\left(x_{i}, y_{j}\right)$.

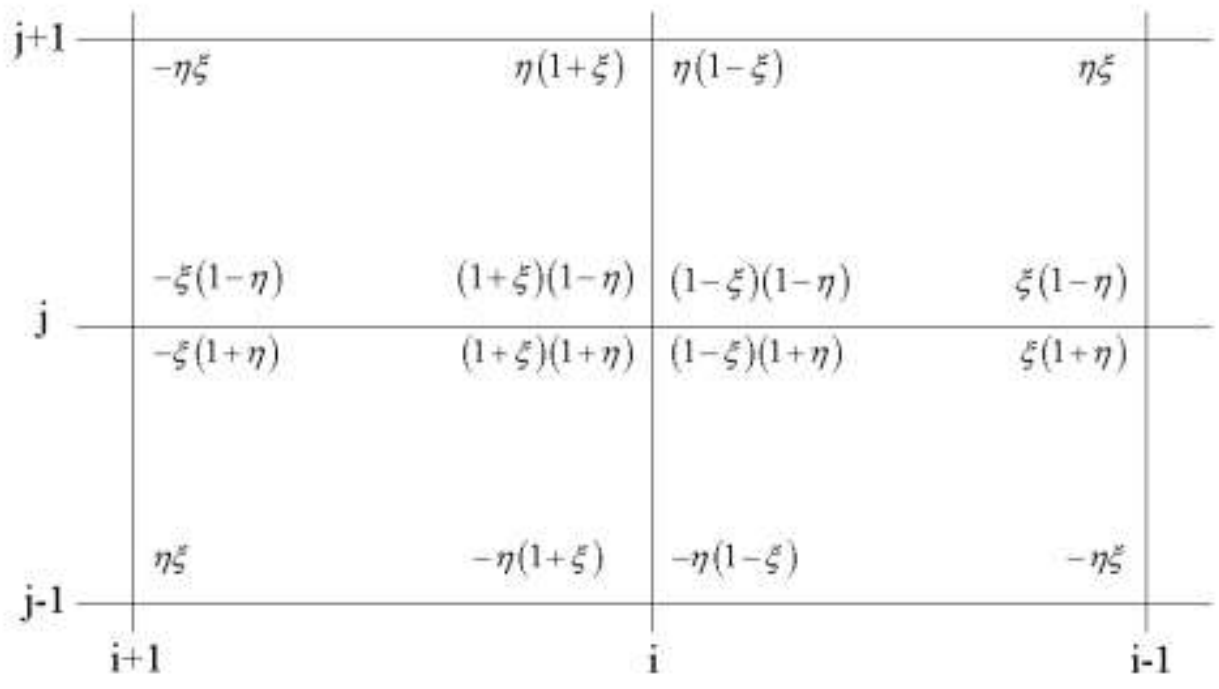

Figure 1: 4 neighborhood normalized elements of arbitrary internal point $\left(x_{i}, y_{j}\right)$

Now, through integral (6), we have three types of blocked tri-diagonal matrices. For the matrix $M_{1}$ we have the matrix elements as figure (2). 


\begin{tabular}{|c|c|}
$\frac{1}{6 h_{x}^{2}}-\frac{1}{3 h_{y}^{2}}$ & $-\frac{1}{6 h_{x}^{2}}-\left.\frac{1}{6 h_{y}^{2}}\right|^{\mathrm{i}+1}$ \\
$\frac{1}{3 h_{x}^{2}}+\frac{1}{3 h_{y}^{2}}$ & $-\frac{1}{3 h_{x}^{2}}+\frac{1}{6 h_{y}^{2}}$ \\
$\mathrm{i}$ & $\mathrm{i}+1$
\end{tabular}

Figure 2: $M_{1}$ matrix elements

and $M_{2}$ matrix elements are as figure (3).

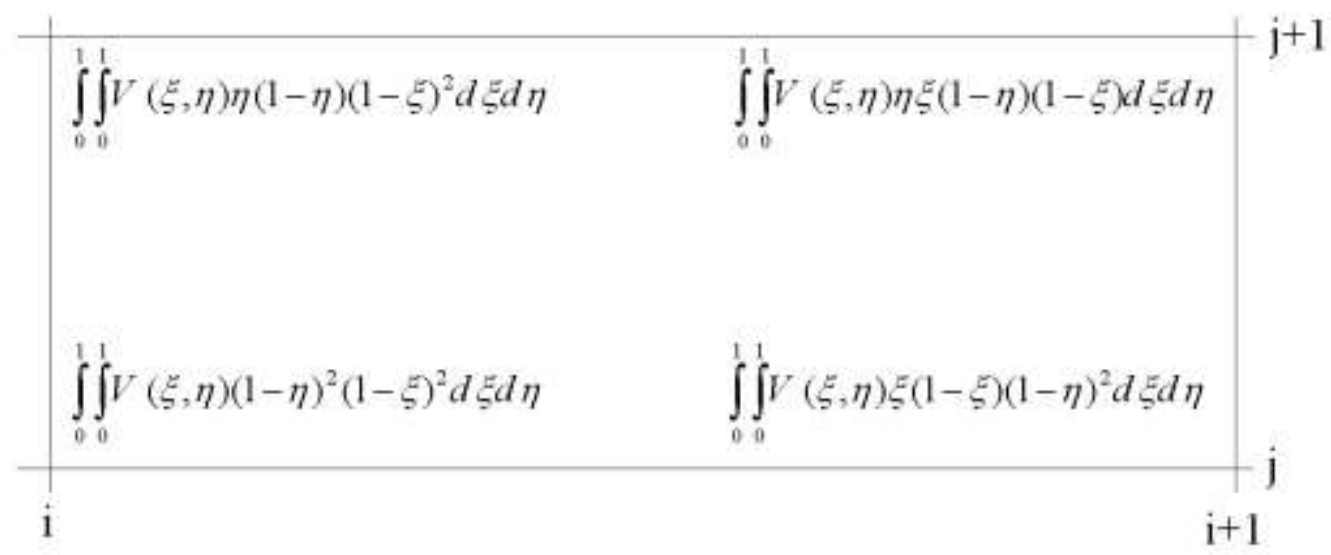

Figure 3: $M_{2}$ matrix elements

And $M_{3}$ matrix elements are as figure (4).

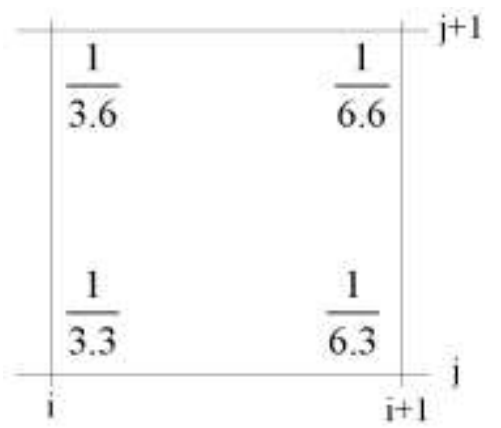

Figure 4: $M_{3}$ matrix elements

When we evaluate the above mentioned sub-matrices, we will have the following eigenvalue equation system, 
$-\alpha M_{1} \psi+M_{2} \psi=E M_{3} \psi$,

where, $E$ are the energy eigenvalues.

\section{Results and Discussions}

By using a finite element method and compact density matrix formalism, we have investigated the electronic properties of multi-abloom nano-flowers. Figure (5) shows the few multi abloom potential profile.
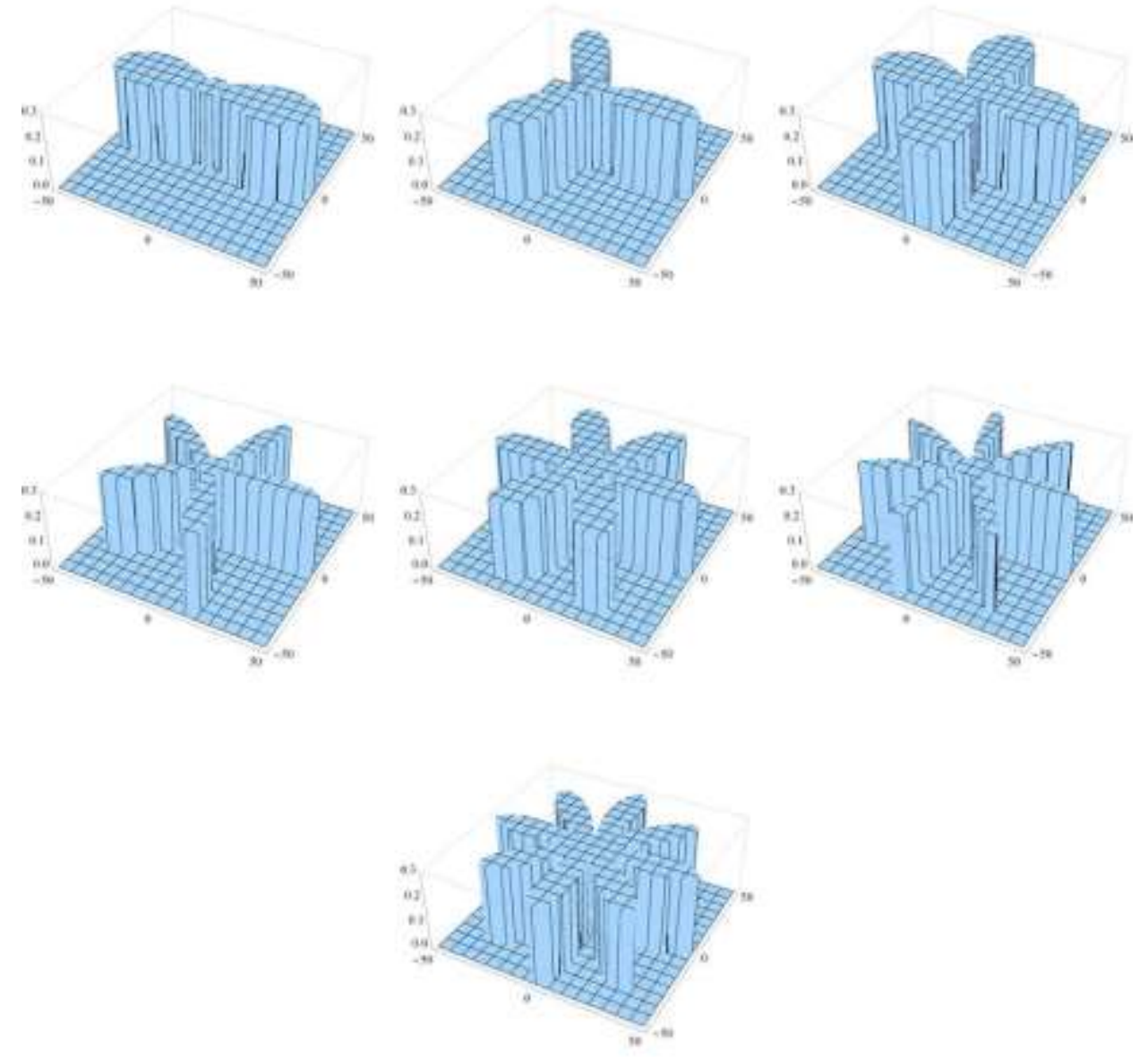

Figure 5: confining potential of the systems with 2 to 8 number of ablooms. ( $r^{2}=\operatorname{Cos}(2 \theta)$ for 2 ablooms, $r=\operatorname{Cos}(3 \theta)$ for 3 ablooms, $r=\operatorname{Cos}(2 \theta)$ for 4 ablooms, and so on.)

In the figure (6) we have presented the variation of the first 20 lowest eigen-energies of a 5 -abloom nano-flower as a function of the domain size. In this figure, we see that, as the domain size increases the energies decreases. 


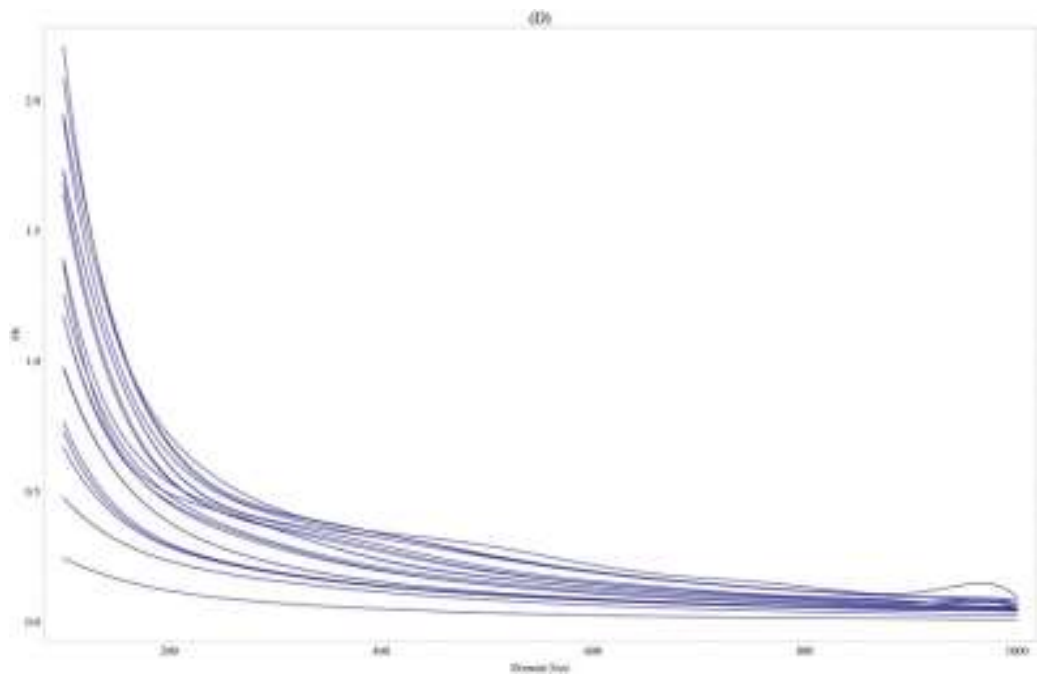

Figure 6: variation of the first 20 lowest eigen-energies as a function of the domain size. Also, in the figure (7), we have given the variation of the first 20 lowest eigen-energies of a 5 -abloom nano-flower as a function of the number of ablooms. Here, we see a nonlinear behavior in the energy values when the number of ablooms increases.

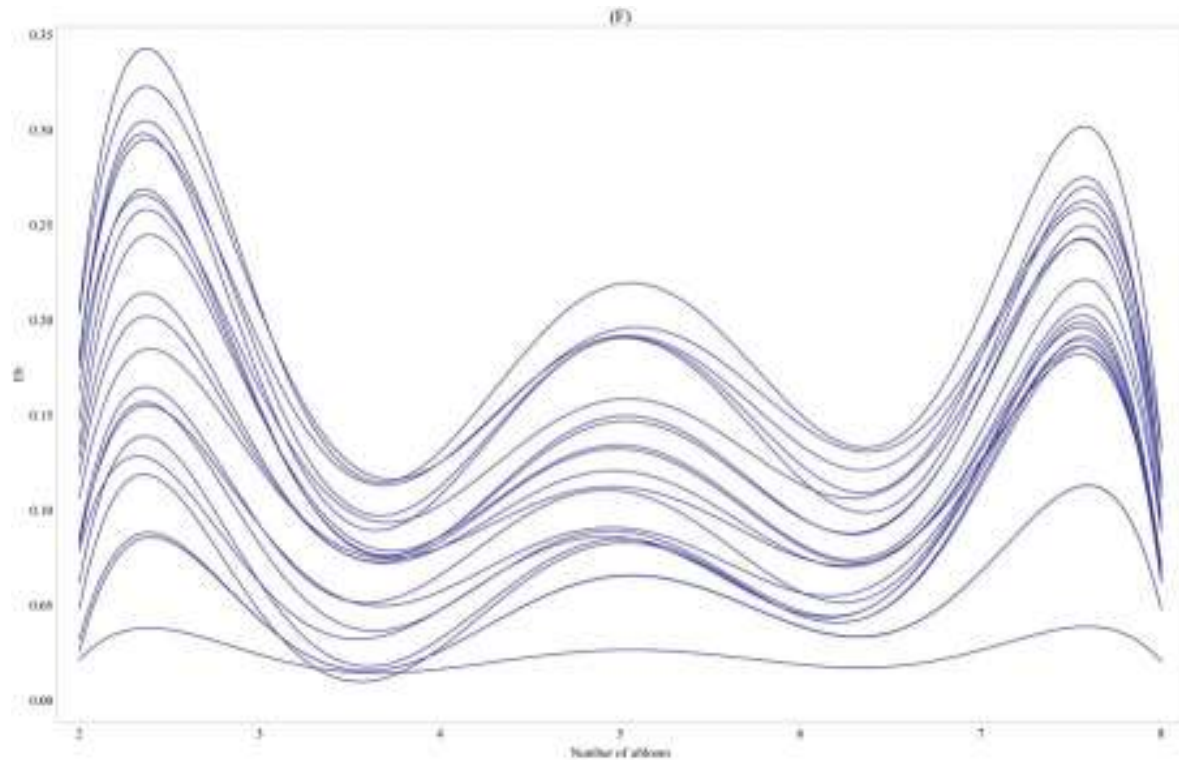

Figure 7: variation of the first 20 lowest eigen-energies as a function of the number of ablooms.

In the next four figures (8-1) to (8-4), we have presented the 9 eigen-functions of the system with $2,3,5$, and 8 number of ablooms. These figures indicate the distribution of the probability densities inside the quantum structure. This probabilities show the probability of finding an electron in an assumed structures and therefore can provide us information about the free careers distribution. 

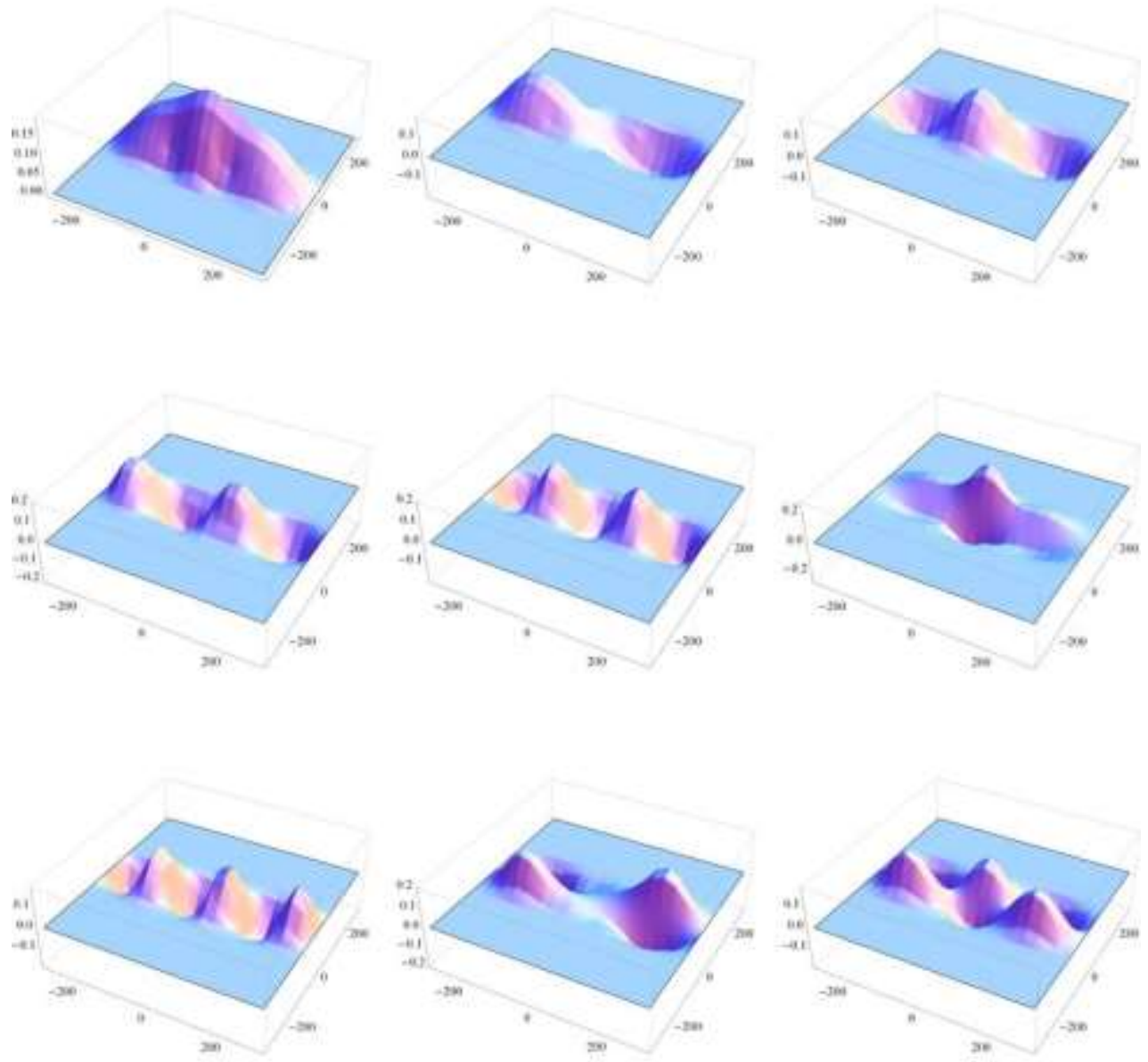

Figure 8-1: First 9 eigen-functions of the system with 2 ablooms. (the 2 ablooms rose can be depicted by $\left.r^{2}=\operatorname{Cos}(2 \theta)\right)$ 

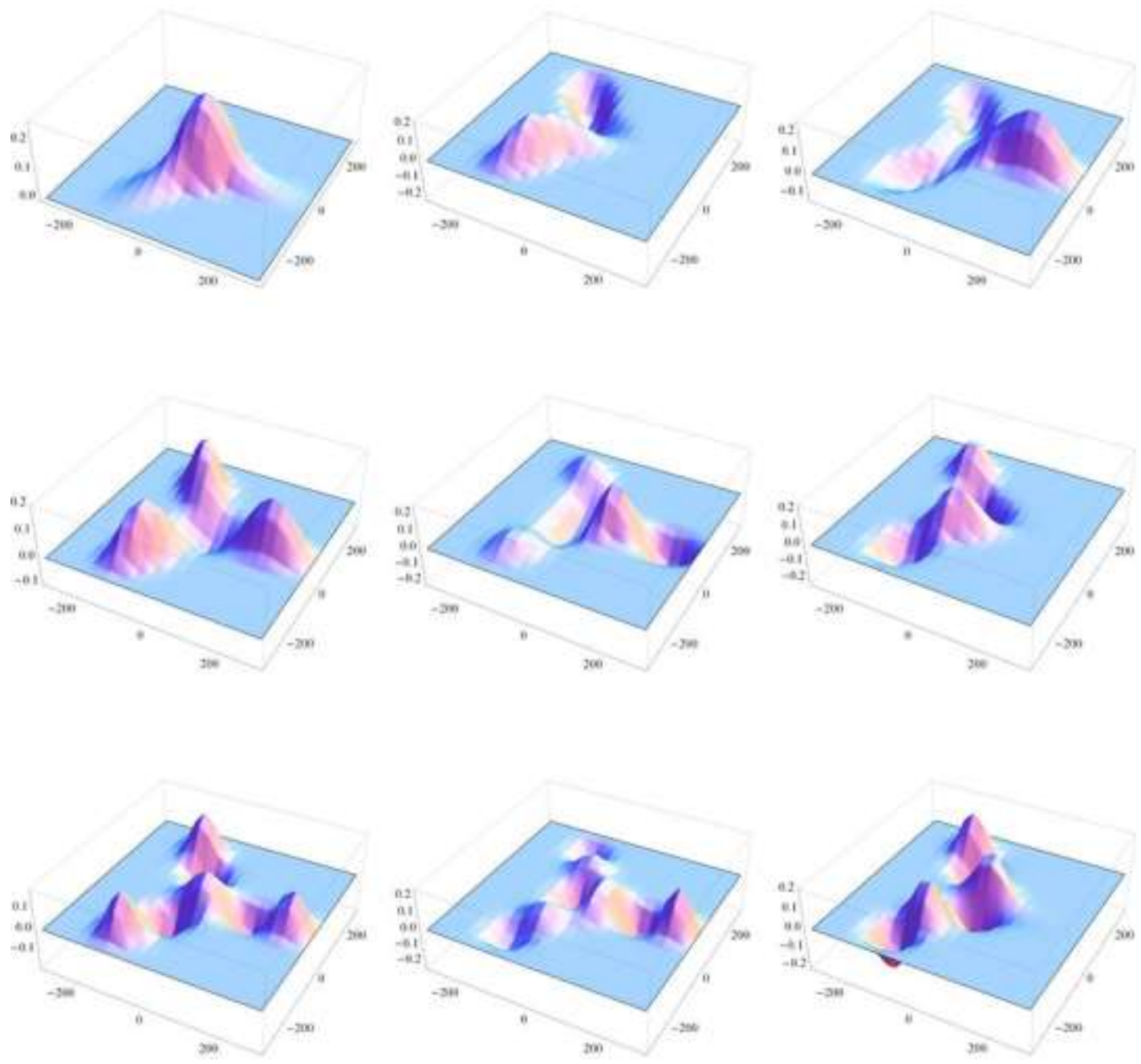

Figure 8-2: First 9 eigen-functions of the system with 3 ablooms. (the 3 ablooms rose can be found by $r=\operatorname{Cos}(3 \theta)$ or $r=\operatorname{Sin}(3 \theta))$ 

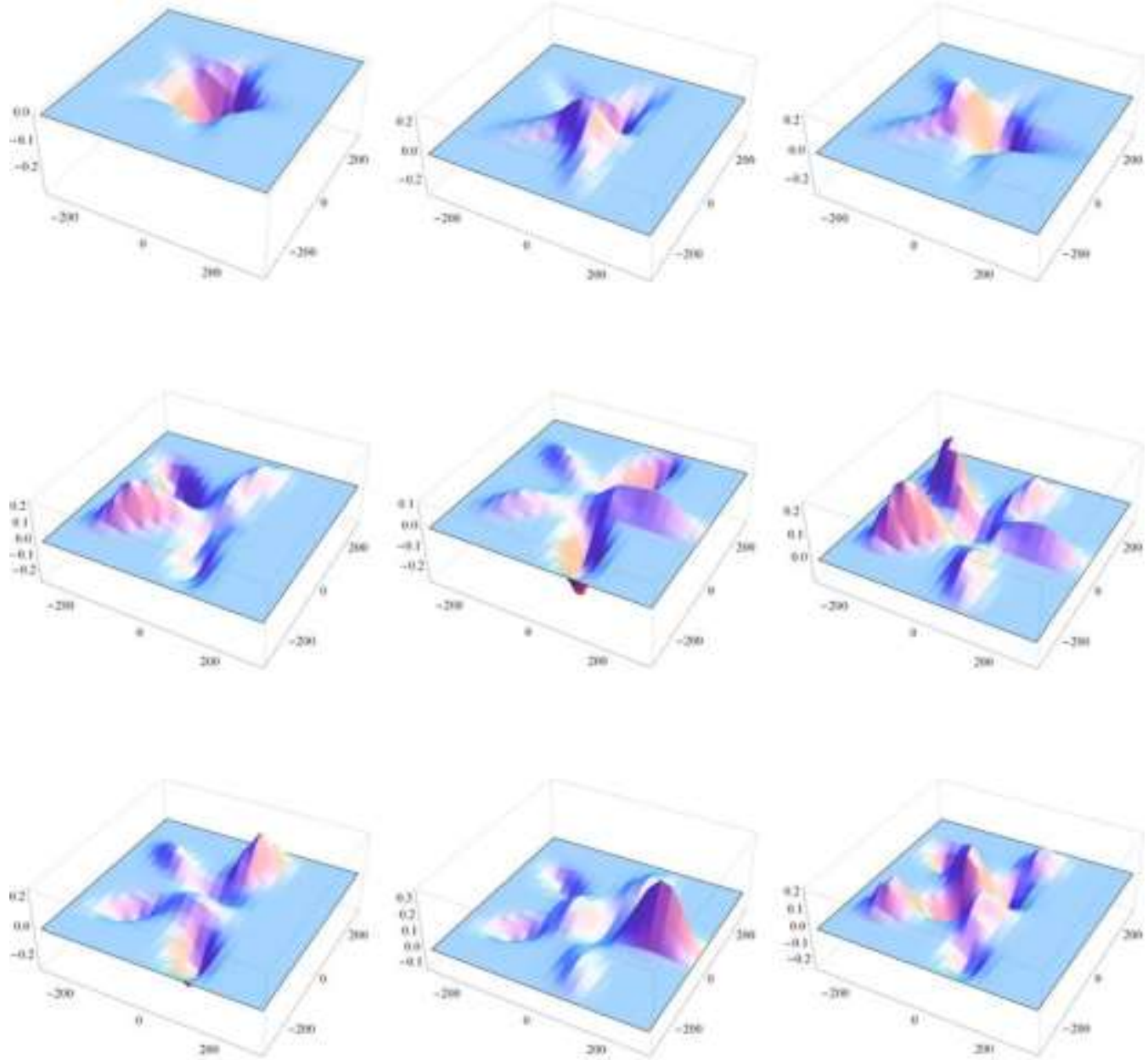

Figure 8-3: First 9 eigen-functions of the system with 5 ablooms. 

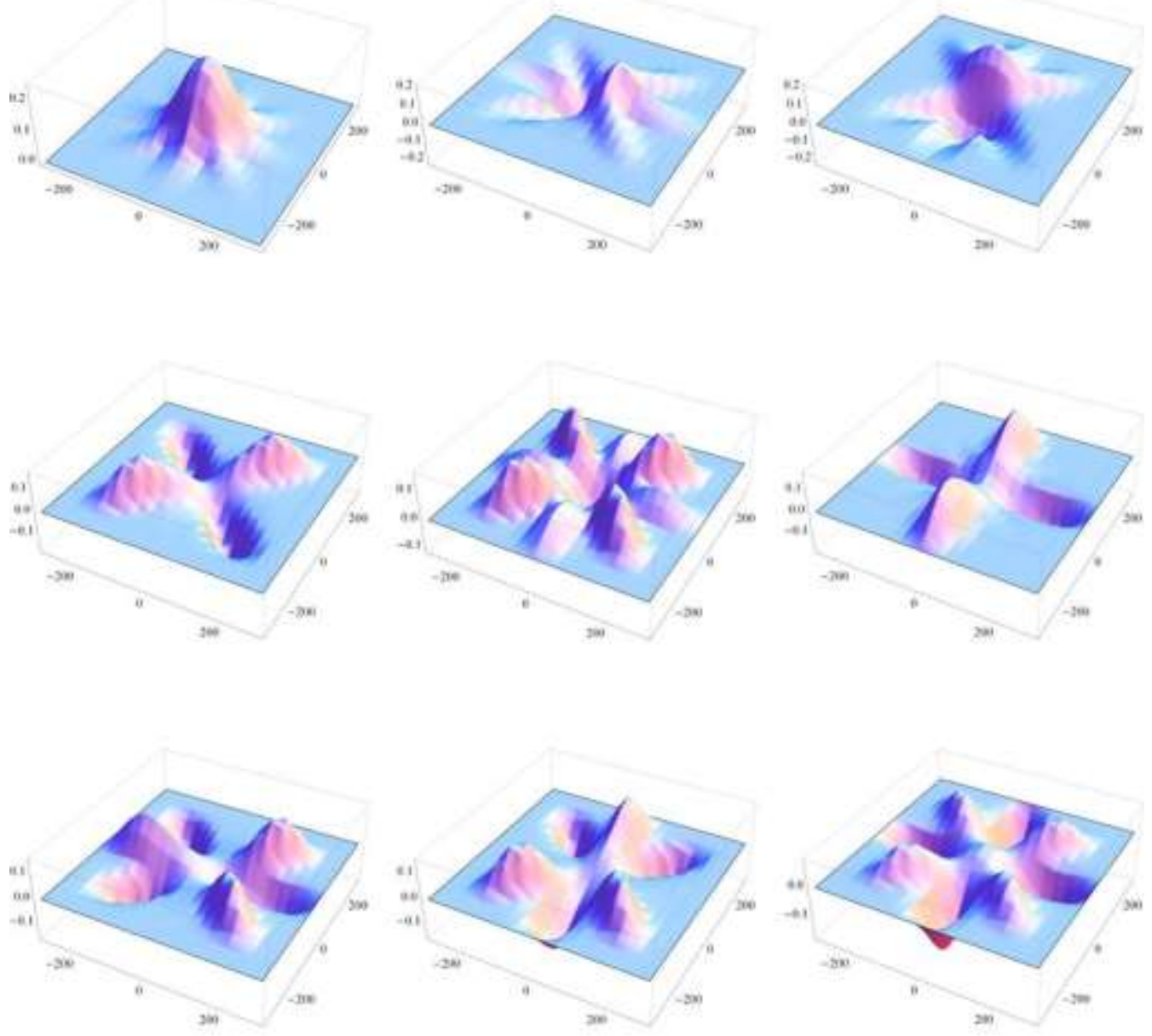

Figure 8-4: First 9 eigen-functions of the system with 8 ablooms.

Figure (8) and (9) also show the effects of the system length and number of ablooms on the dipole matrix elements $M_{21}=\int_{a_{y}}^{b_{y}} \int_{a_{x}}^{b_{x}}|x| \psi_{1}(x, y) \psi_{2}(x, y) d x d y$, respectively. As figure (8) shows, when the system length increases, the dipole matrix elements $M_{21}$ also increases. However, the figure (9) indicates that the variation of the dipole matrix elements $M_{21}$ as a function of the number of ablooms is an oscillatory function. 


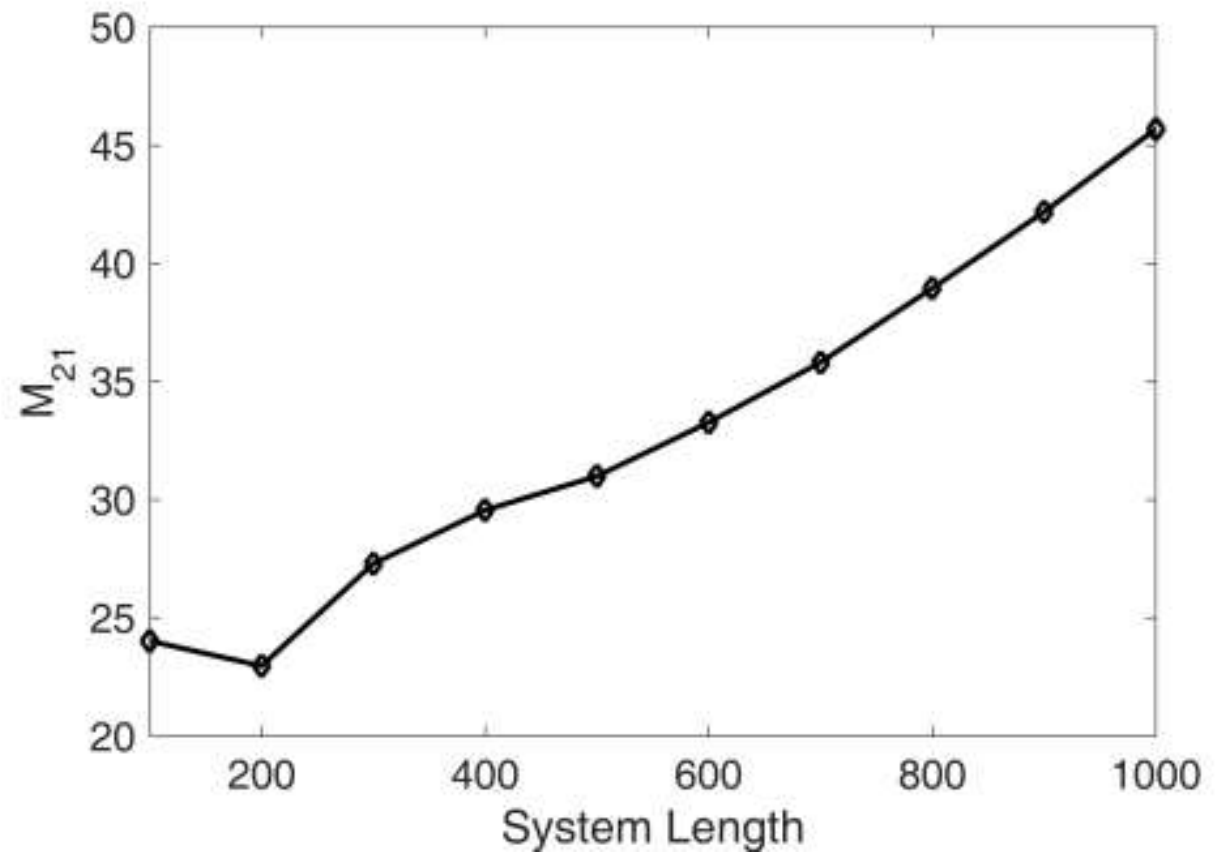

Figure 8: variation of the dipole matrix elements $M_{21}$ as a function of the system length.

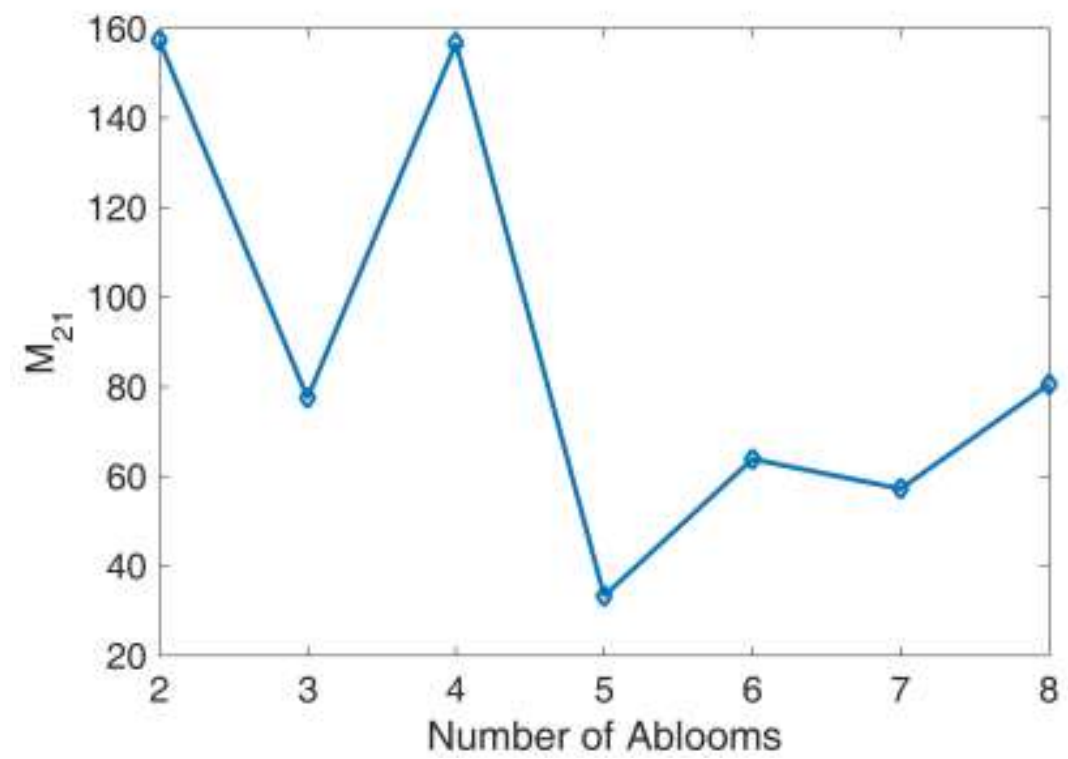

Figure 9: variation of the dipole matrix elements $M_{21}$ as a function of the number of ablooms.

\section{Conclusion}

In this work, we investigated the electronic properties of multi-abloom nanoflower. By using detailed finite element method, we showed that as the domain size increased, the energies decreased. A nonlinear behavior in the energy values observed 
when the number of ablooms increased. When the system length increased, the dipole matrix elements $M_{21}$ also increased. The variation of the dipole matrix elements $M_{21}$ as a function of the number of ablooms was an oscillatory function.

\section{References}

[1] Trinidad García, Francisco Manuel Gómez-Campos, and Salvador Rodríguez-Bolívar, "Influence on miniband structure of size variations in regimented InAs/GaAs quantum dots arrays", Journal of Applied Physics 114, 064311 (2013).

[2] R.V.N. Melnik and M. Willatzen, "Bandstructures of conical quantum dots with wetting layers", Nanotechnology 15, 1(2004).

[3] Guanghui Zhou and Yuan Li, "Electromagnetic-field-induced resonant structures for an open rectangular quantum dot", Eur. Phys. J. B 46, 127 (2005).

[4] Gabriel Bester and Alex Zunger, "Cylindrically shaped zinc-blende semiconductor quantum dots do not have cylindrical symmetry: Atomistic symmetry, atomic relaxation, and piezoelectric effects", Physical Review B 71, 045318 (2005).

[5] Ruihao Wei, Wenfang Xie, "Optical absorption of a hydrogenic impurity in a discshaped quantum dot", Current Applied Physics 10, 757 (2010).

[6] E. Sadeghi, F. Vahdatnejad, M. Moradi LM, "Effect of polarization charges on impurity binding energy in elliptical quantum wire", Superlattices and Microstructures 58, 165 (2013).

[7] A.H. Rodriguez and H.Y. Ramırez, "Analytical calculation of eigen-energies for lensshaped quantum dot with finite barriers", Eur. Phys. J. B 66, 235 (2008).

[8] Tsung-Min Hwang a, Wen-Wei Lin b, Wei-Cheng Wang b, Weichung Wang, "Numerical simulation of three dimensional pyramid quantum dot", Journal of Computational Physics 196, 208 (2004).

[9] Chayanika Bose, "Binding energy of impurity states in spherical quantum dots with parabolic confinement", Journal of Applied Physics, 83, 3089 (1998).

[10] B. H. Wu and J. C. Cao, "Interference of conductance and shot noise properties of photon-assisted transport through a T-shaped double quantum dot", PHYSICAL REVIEW B 73, 205318 (2006).

[11] Tie-Feng Fang and Shun-Jin Wang, "Cross correlations and shot noise in a Y-shaped quantum dot", J. Phys.: Condens. Matter 19, 026204 (2007) 
[12] Physica E 66107 (2015).

[13] A. V. Nenashev, and A. V. Dvurechenskii, "Variational method of energy level calculation in pyramidal quantum dots", J. Appl. Phys. 127, 154301 (2020).

[14] Mohammad El-Said, "Spectroscopic structure of two interacting electrons in a quantum dot by the shifted 1/N expansion method", PHYSICAL REVIEW B 61, 13026 (2000).

[15] M. Governale, M. Macucci, G. Iannaccone, C. Ungarelli, and J. Martorell, "Modeling and manufacturability assessment of bistable quantum-dot cells", J. Appl. Phys. 85, 2962 (1999).

[16] Shintaro Nomura, Yoshinobu Aoyagi, "Density of states of a quantum dot array probed by photoluminescence spectra", Surface Science 529, 171 (2003).

[17] A. D. Gu“c slü, Qing Feng Sun, Hong Guo, and R. Harris, "Geometric blockade in a quantum dot: Transport properties by exact diagonalization", Physical Review B 66, 195327 (2002).

[18] Arpan Deyasi, S. Bhattacharyya b, N.R. Das, "Computation of intersubband transition energy in normal and inverted core-shell quantum dots using finite difference technique", Superlattices and Microstructures 60, 414 (2013).

[18] V.A. Shuvayev, L.I. Deych, I.V. Ponomarev, A.A. Lisyansky, "Self-consistent Hartree method for calculations of exciton binding energy in quantum wells", Superlattices and Microstructures 40, 77 (2006).

[19] O. Stier, M. Grundmann, and D. Bimberg, "Electronic and optical properties of strained quantum dots modeled by 8-band k.p theory", Physical Review B 59, 5688 (1999).

[20] A. D. Güçlü, C. J. Umrigar, "Maximum-density droplet to lower-density droplet transition in quantum dots", Physical Review B 72, 045309 (2005).

[21]V Golubnychiy, H Baumgartner, M Bonitz, A Filinov and H Fehske, "Screened Coulomb balls_-structural properties and melting behavior", J. Phys. A: Math. Gen. 39, 4527 (2006).

[22] M. A. Naser, M. J. Deen, and D. A. Thompson, "Spectral function and responsivity of resonant tunneling and superlattice quantum dot infrared photodetectors using Green's function", Journal of Applied Physics 102, 083108 (2007). 
[23] Sameer M. Ikhdair, Majid Hamzavi, "A quantum pseudodot system with twodimensional pseudoharmonic oscillator in external magnetic and Aharonov-Bohm fields", Physica B 407, 4198 (2012).

[24] Mohammad Mardaania, Ali A. Shokri, Keivan Esfarjani, "Analytical results on coherent conductance in a general periodic quantum dot: Transfer matrix method", Physica E 28, 150 (2005).

[25] Chenxing Zhao \& Yifeng E. \& Louzhen Fan, "Enhanced electrochemical evolution of oxygen by using nanoflowers made from a gold and iridium oxide composite", Microchim Acta 178, 107 (2012).

[26] Daniel M. Cunha, Flavio L. Souza, "Facile synthetic route for producing onedimensional zinc oxide nanoflowers and characterization of their optical properties", Journal of Alloys and Compounds 577, (1582013).

[27] Yong-Jin Kim, Jinkyoung Yoo, Byoung-Hwa Kwon, Young Joon Hong, Chul-Ho Lee and Gyu-Chul $\mathrm{Yi}$, "Position-controlled $\mathrm{ZnO}$ nanoflower arrays grown on glass substrates for electron emitter application", Nanotechnology 19, 315202 (2008).

[28] Liming Song, Anatolii Lukianov, Denys Butenko, Haibo Li, Junkai Zhang, Ming Feng3, Liying Liu, Duo Chen and N. I. Klyui, "Facile Synthesis of Hierarchical Tin Oxide Nanoflowers with Ultra-High Methanol Gas Sensing at Low Working Temperature", Nanoscale Research Letters 14, 84 (2019).

[29] Pavel Šolín, "Partial Differential Equations and the Finite Element Method", 2005, John Wiley \& Sons.

[30] Dietrich Braess, "Finite Elements, Theory, Fast Solvers, and Applications in Elasticity Theory”, Cambridge Univ. Press, 2007.

[31] J. T. Marti, "Introduction to Sobolev spaces and finite element solution of elliptic boundary value problems", Academic Press, 1986.

[32] A. R. Mitchell and R. Wait, "Finite Element Analysis and Applications", John Wiley, 1985.

[33] J. N. Reddy, “An Introduction to the Finite Element Method”, Mc Graw-Hill, 2005.

[34] S. Brenner, R. Scott, "The Mathematical Theory of Finite Element Methods", Springer, 2007.

[35] B. A. Finlayson, “The Method of Weighted Residuals and Variational Principles, with application in fluid mechanics, heat and mass transfer", Academic Press, 1972. 
[36] W. F. Ames, "Numerical Methods for Partial Differential Equations, 3Ed.", Academic Press, 1992.

[37] P. K. Chattopadhyay, "Mathematical Physics", New Age Int. (P) Ltd., 1990. 
Figures

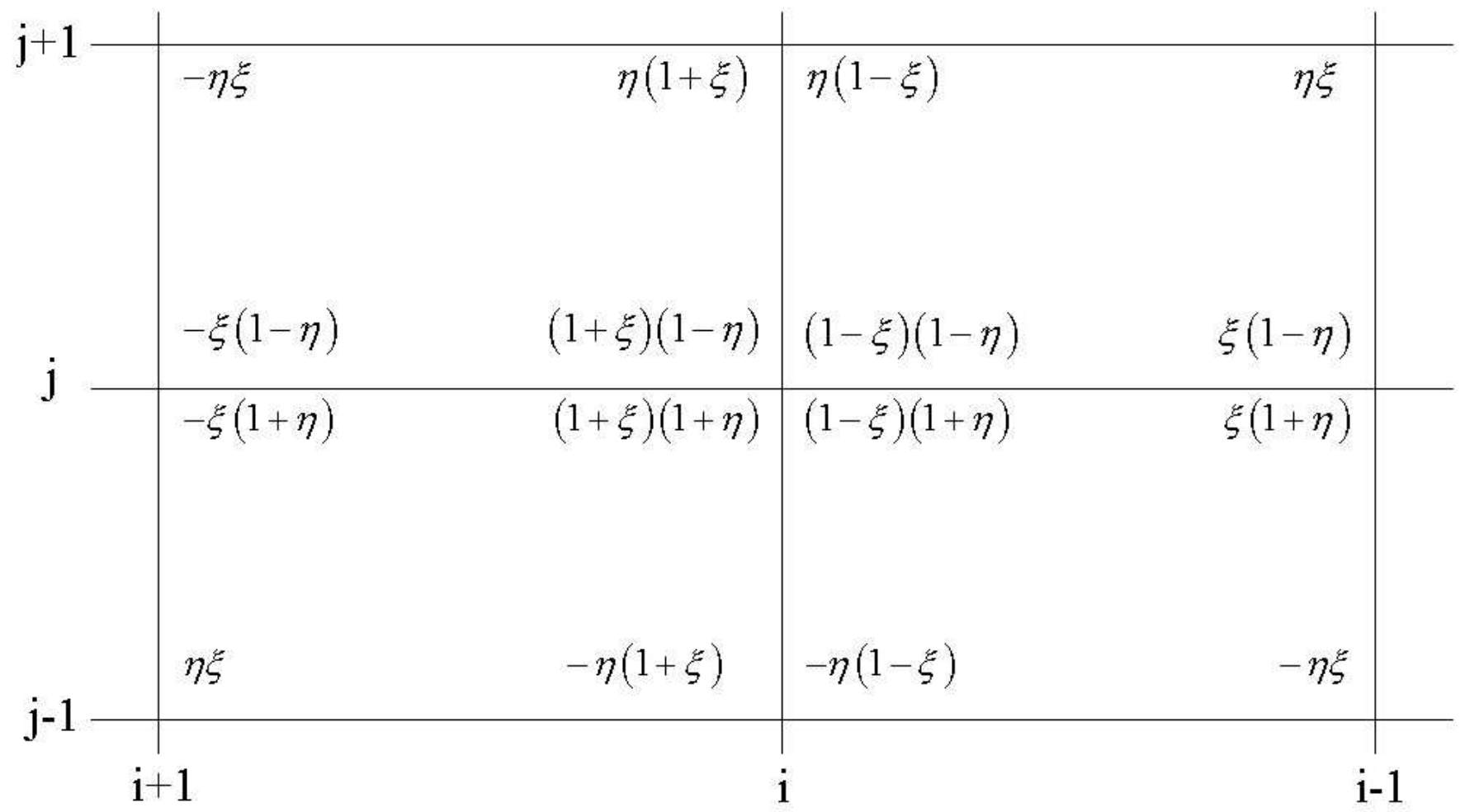

\section{Figure 1}

See the Manuscript Files section for the complete figure caption. 


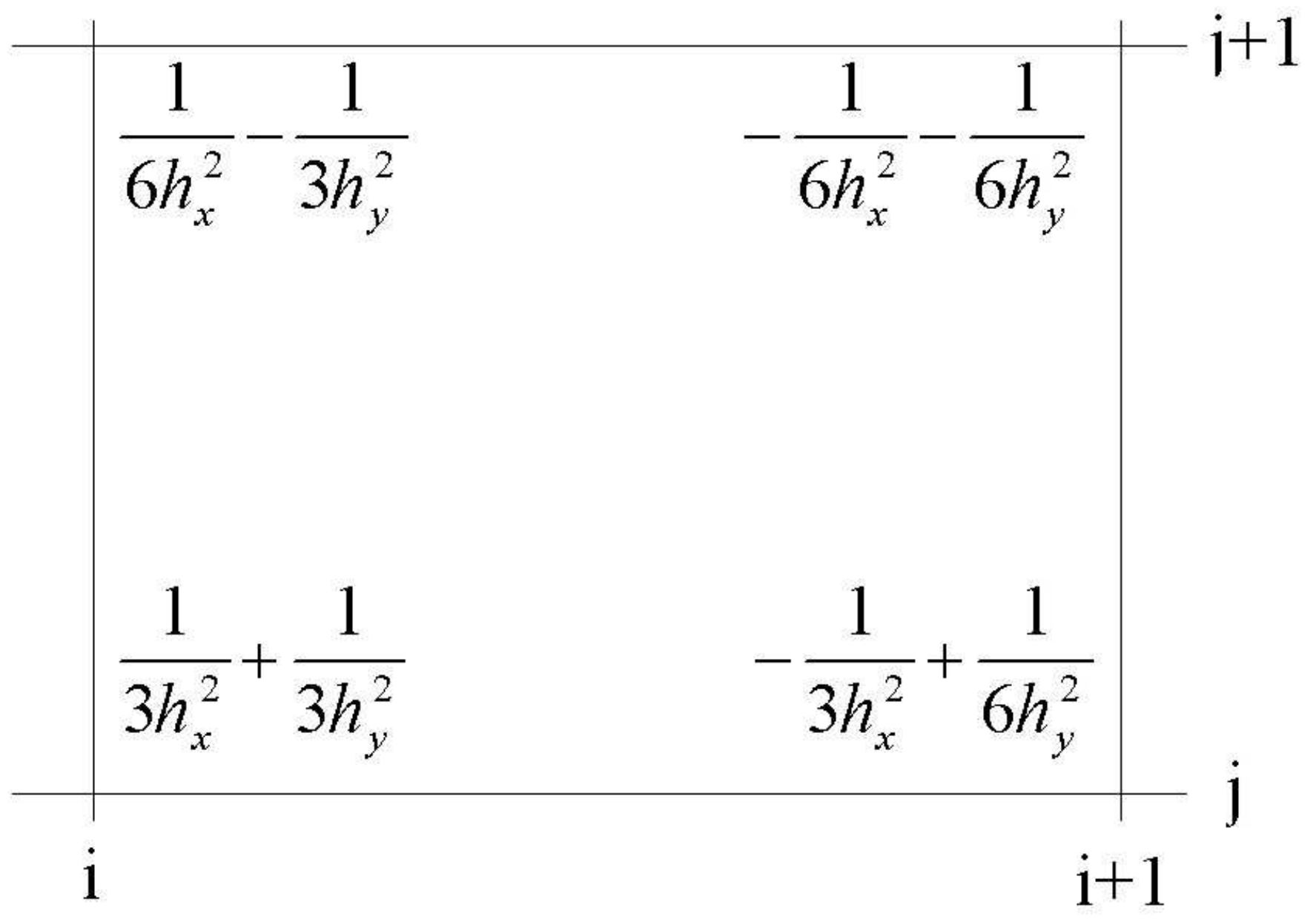

Figure 2

See the Manuscript Files section for the complete figure caption. 


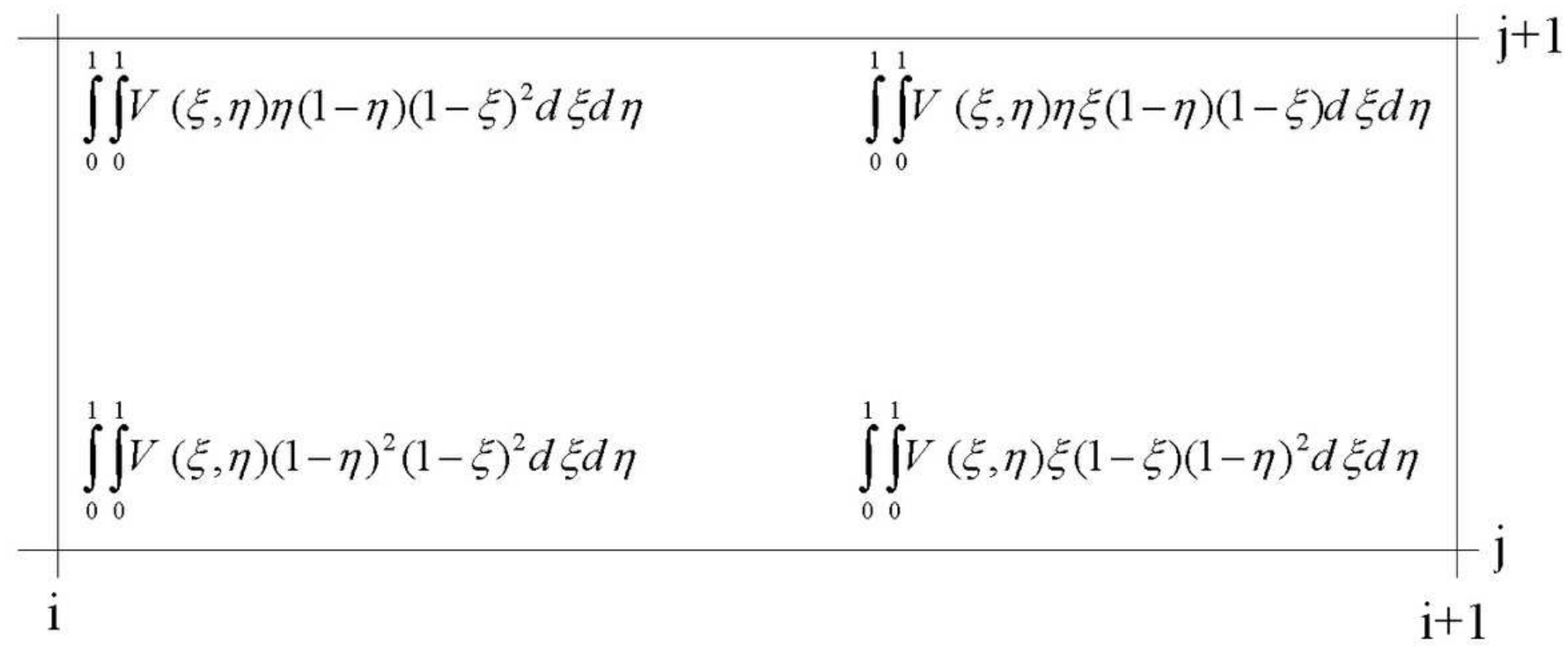

Figure 3

See the Manuscript Files section for the complete figure caption. 


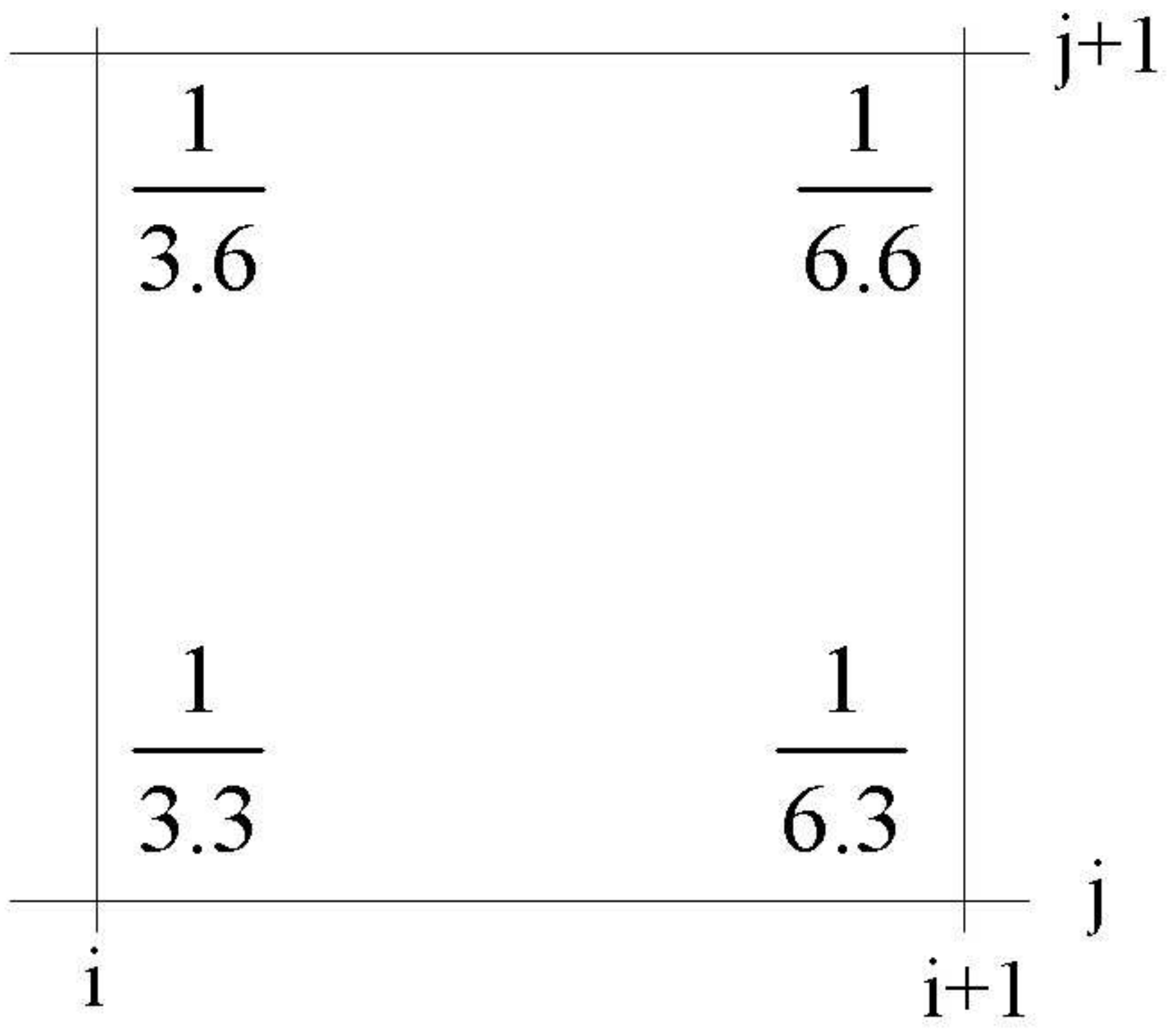

Figure 4

See the Manuscript Files section for the complete figure caption. 



Figure 5

See the Manuscript Files section for the complete figure caption. 
(D)

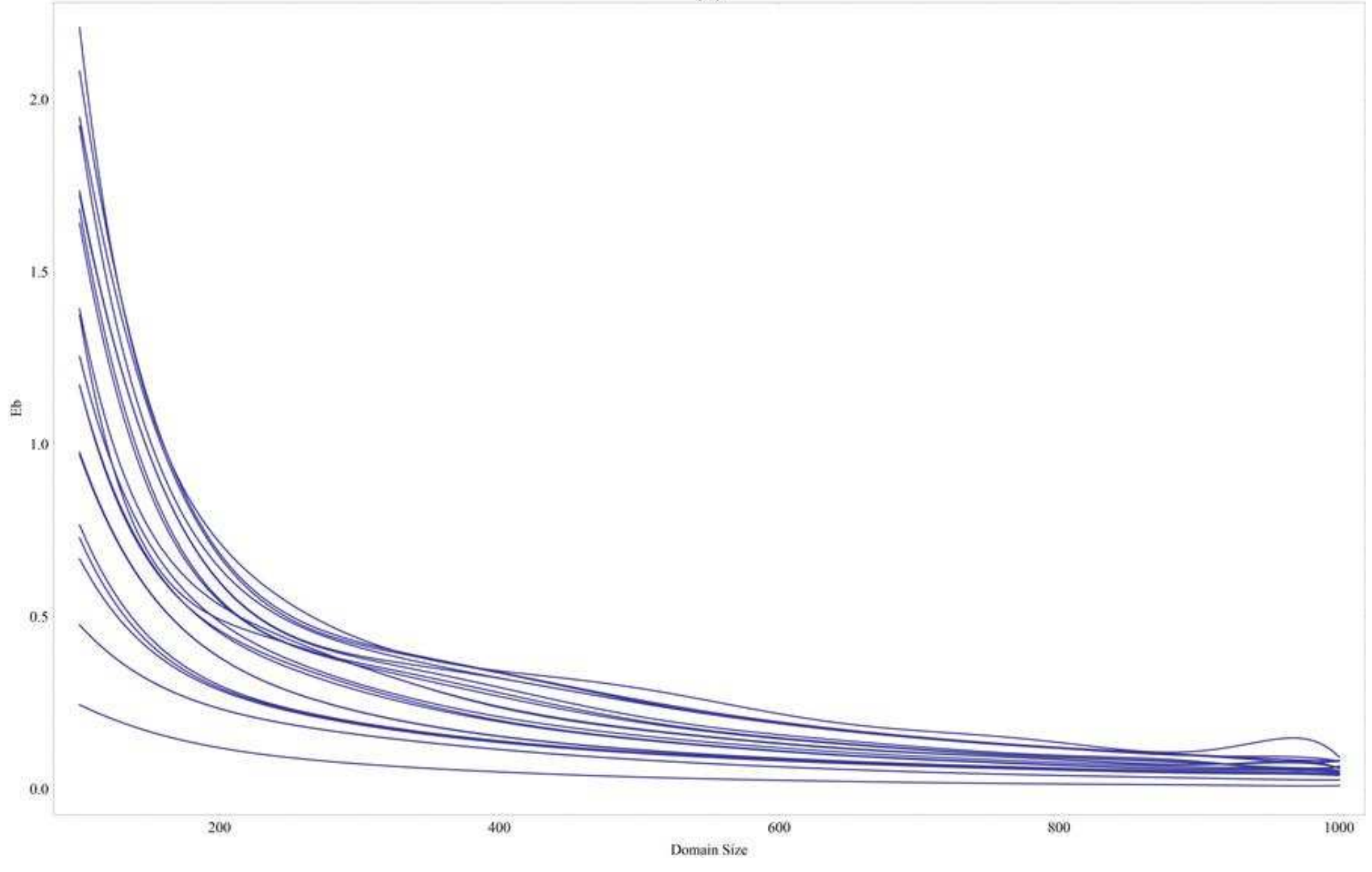

Figure 6

See the Manuscript Files section for the complete figure caption. 


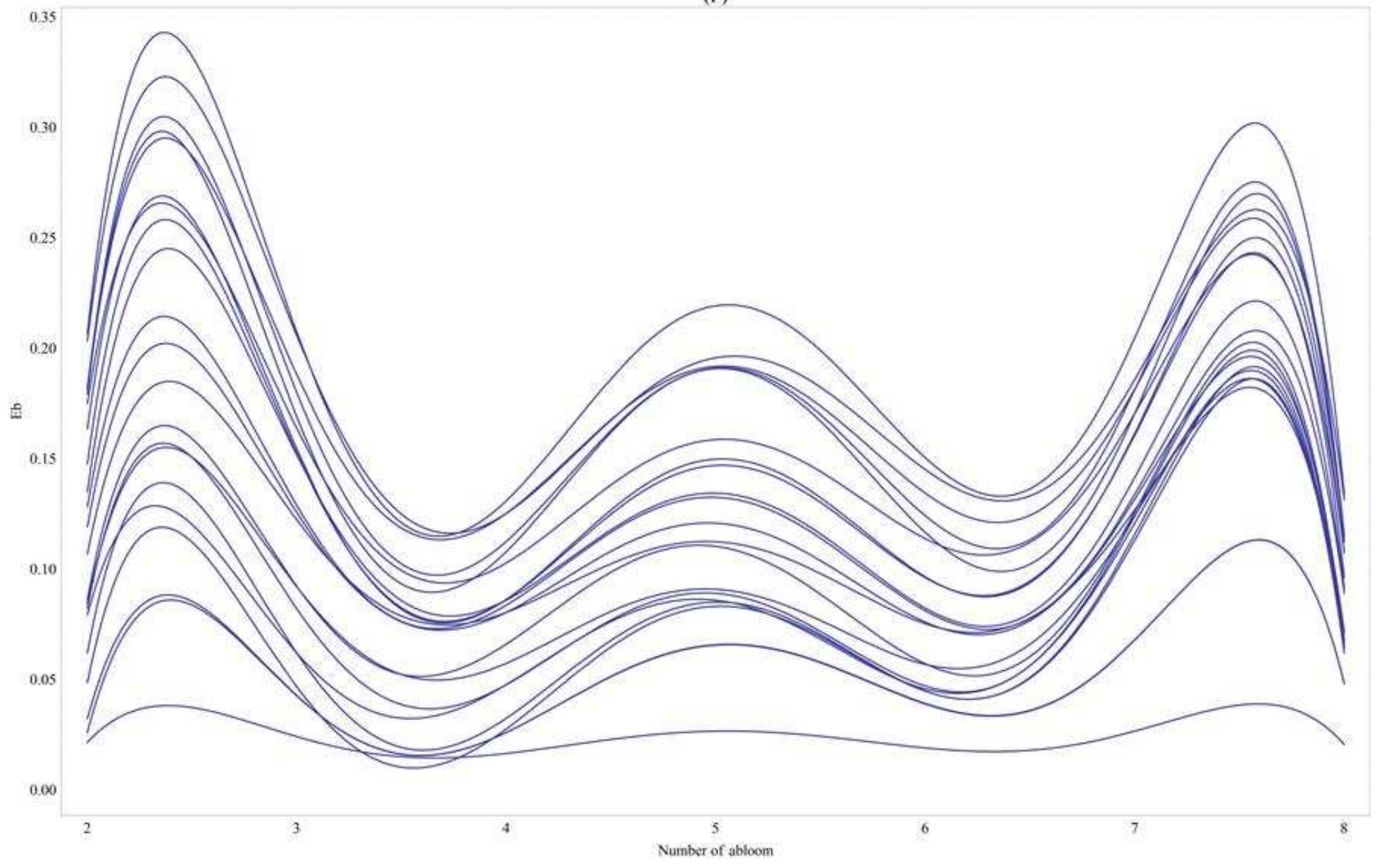

Figure 7

See the Manuscript Files section for the complete figure caption. 

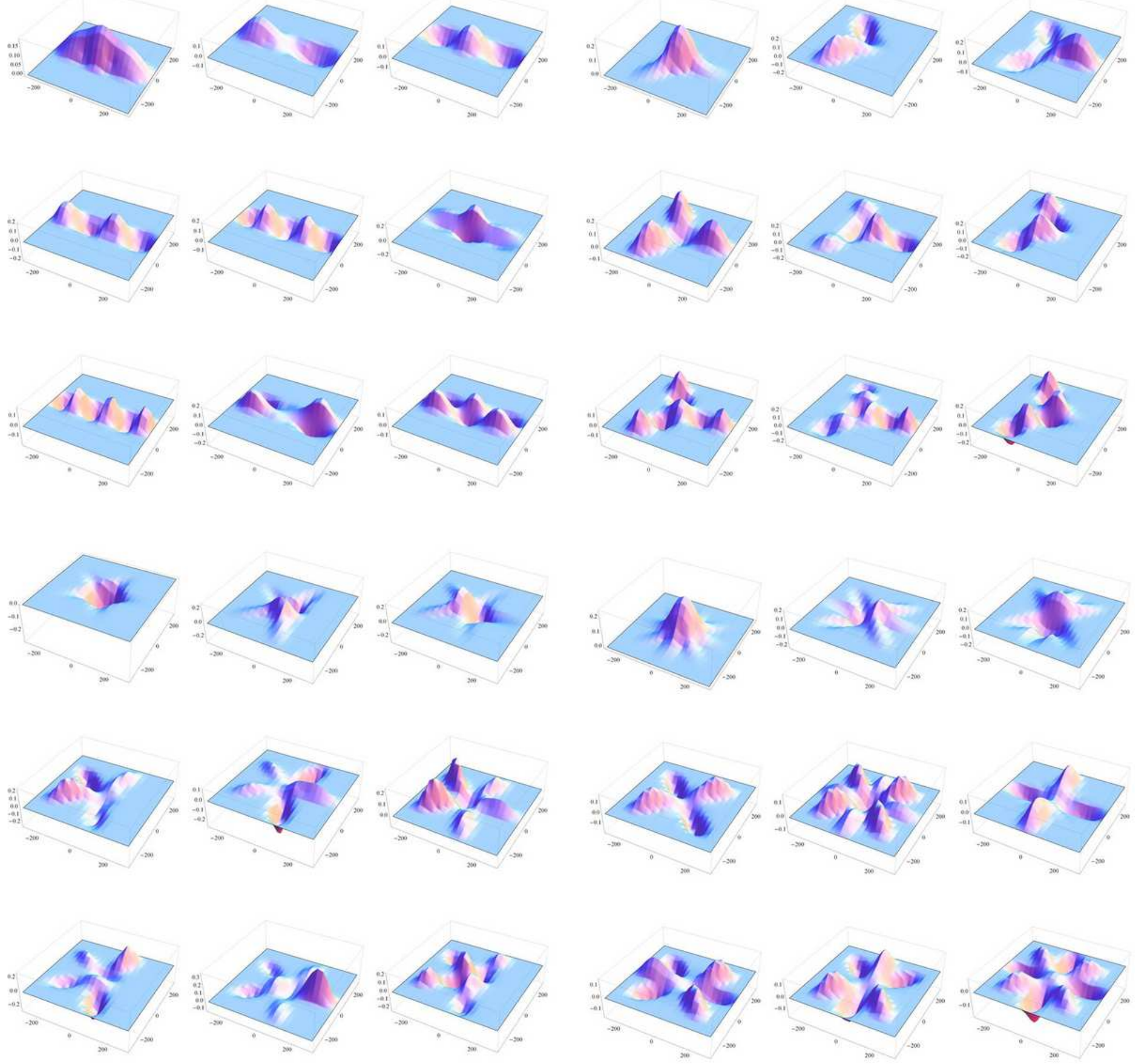

Figure 8

See the Manuscript Files section for the complete figure caption. 


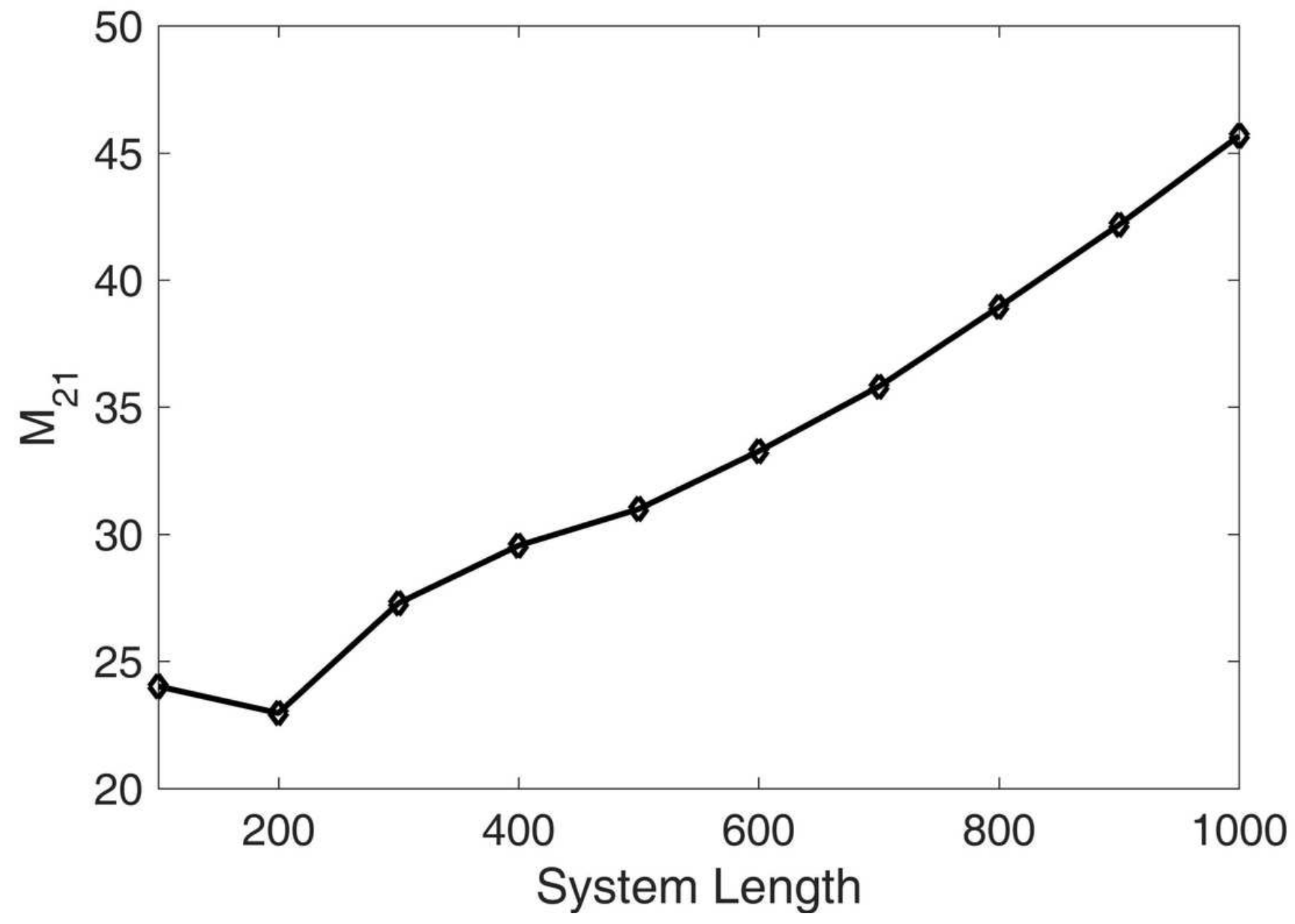

Figure 9

See the Manuscript Files section for the complete figure caption. 


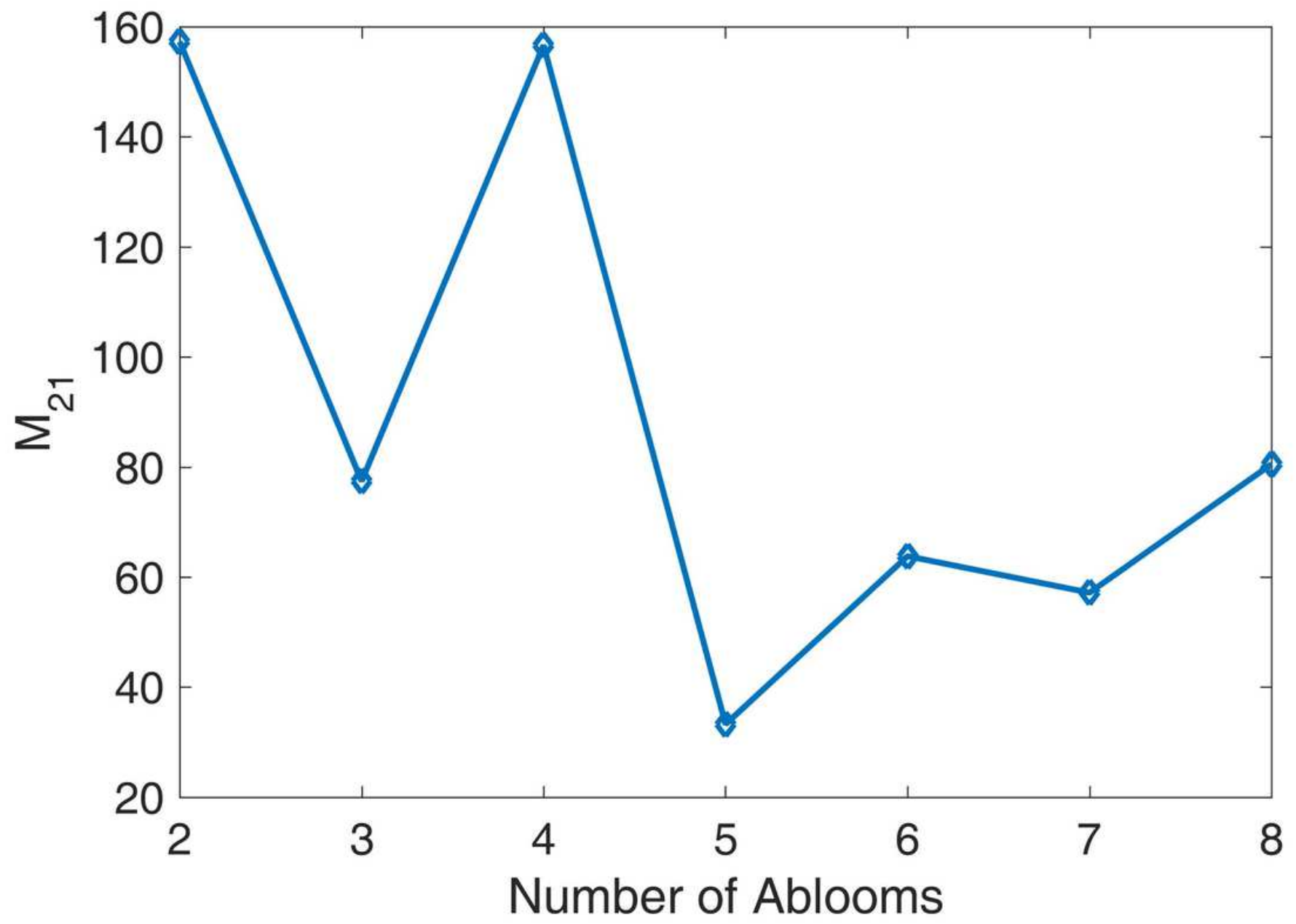

Figure 10

See the Manuscript Files section for the complete figure caption. 